\title{
Signals in Macaque Striate Cortical Neurons that Support the Perception of Glass Patterns
}

\author{
Matthew A. Smith, ${ }^{1}$ Wyeth Bair, ${ }^{1,2}$ and J. Anthony Movshon ${ }^{1,2}$ \\ ${ }^{1}$ Center for Neural Science and ${ }^{2 H}$ Howard Hughes Medical Institute, New York University, New York, New York 10003
}

Glass patterns are texture stimuli made by pairing randomly placed dots with partners at specific offsets. The strong percept of global form that arises from the sparse local orientation cues has made these patterns the subject of psychophysical investigations, yet neuronal responses to Glass patterns have not been studied. We measured the responses of neurons in macaque striate cortex (V1) to dynamic, translational Glass patterns as a function of dot separation and dot-pair orientation. Responses were selective, but were on average more than an order of magnitude weaker than responses to sinusoidal gratings. Response and selectivity were greatest when the dot-pair orientation matched that of the preferred grating and when dot separation was between one-quarter and one-half of the spatial period of the optimal grating; changing the dot-pair separation or inverting the contrast of one of the dots radically changed the orientation selectivity. We computed the expected responses for a receptive field model to translational Glass patterns and found that the complexity of our V1 tuning curves could be understood in terms of the responses of linear filters to pairs of dots. This modeling connects our understanding of V1 receptive fields as rectified, quasi-linear filters with results from psychophysical studies of Glass patterns. Our results provide a basis for studying how subsequent visual areas integrate weak, local signals into global form percepts.

Key words: Glass patterns; macaque monkey; primary visual cortex; V1; random dots; orientation selectivity; linear filter; spatial frequency
Glass patterns (Glass, 1969; Glass and Perez, 1973) have been used in numerous psychophysical studies to probe form-detecting mechanisms in human observers (Glass and Switkes, 1976; DeValois and Switkes, 1980; Prazdny, 1984; Earle, 1985; Prazdny, 1986; Dakin, 1997a; Wilson et al., 1997; Wilson and Wilkinson, 1998; Ross et al., 2000; Dakin and Bex, 2001). These patterns are created by taking a "seed" pattern of randomly placed dots and then pairing each dot with another according to a particular geometric rule. The example Glass patterns shown on the left side of Figure 1 were generated by translating, rotating, and magnifying the seed pattern and adding the result back to the original field. The percept of global form in each case is clear, but these global percepts arise purely from the local orientation cues given by pairs of dots. In his first description of these patterns, Glass (1969) speculated on the nature of the cortical responses that they evoked, and proposed that they would be useful for studying the neural basis of form perception. Closely related random-dot stimuli have been used to successfully probe the neuronal mechanisms underlying global coherent percepts in motion processing (Newsome et al., 1989) and depth perception (Poggio et al., 1985).

Consideration of the structure of Glass patterns suggests that they are processed in two stages. The first stage must identify local orientation cues in the otherwise random pattern, and the

Received May 10, 2002; revised July 10, 2002; accepted July 11, 2002.

This work was supported by the Howard Hughes Medical Institute and by a research grant to J.A.M. from the National Institutes of Health (EY02017). M.A.S. was supported in part by a National Eye Institute Institutional Training Grant (T32-7136), and W.B. was supported in part by a grant from the Alfred P. Sloan Foundation. Adam Kohn provided helpf ul comments on this manuscript and, along with James R. Cavanaugh and Najib Majaj, assisted with some of the data collection. Suzanne Fenstemaker provided assistance with histology.

Correspondence should be addressed to J. Anthony Movshon, Center for Neural Science, New York University, 4 Washington Place, Room 809, New York, NY 10003. E-mail: movshon@nyu.edu.

Copyright (C) 2002 Society for Neuroscience $\quad 0270-6474 / 02 / 228334-12 \$ 15.00 / 0$ second stage must combine those local signals to extract largerscale global structures. The local cues for orientation in Glass patterns are individually quite weak, because each dot pair is embedded in a random noisy background. The absence of strong local contours means that the first stage of orientation-selective cells in the cortex might provide sparse, irregular signals; knowledge of these signals is a prerequisite for studying their integration by neurons tuned for global form.

In this paper we report on the responses of neurons in striate cortex (V1), the earliest neurons in the visual pathway with the orientation selectivity needed to begin to parse Glass patterns. V1 receptive fields are quite small compared with the Glass patterns typically used in perceptual experiments and so would typically contain only a small part of the pattern, as demonstrated by the square apertures in Figure 1. A small aperture over any type of extended Glass pattern is approximated well by a translational pattern. We therefore reasoned that a first account of Glass pattern responses in $\mathrm{V} 1$ could be obtained by studying translational patterns like the one in the top panels of Figure 1.

We also simulated the response of V1 neurons to such Glass patterns and derived the response of an oriented filter to arbitrary translational Glass patterns. Simulations of rectified, linear spatial receptive fields (Movshon et al., 1978a,b; DeValois et al., 1982) predict a rather complicated variation in selectivity and responsiveness as a function of dot-pair orientation, separation, and contrast, and they show how receptive field size and aspect ratio can dramatically change selectivity. Recordings of macaque V1 responses to Glass patterns show all the essential features predicted by the model. These results provide a foundation for studying the integration of local signals in downstream visual areas and also offer an account for some psychophysical observations that appear to depend on this first stage of encoding. 


\section{MATERIALS AND METHODS}

Electrophysiology. We recorded extracellularly from single units in primary visual cortex of 12 Cynomolgus macaques (Macaca fascicularis) and 1 pig-tailed macaque (M. nemestrina), ranging in weight from 3.0 to $5.0 \mathrm{~kg}$.

The techniques used in our laboratory for recording from the visual cortex of anesthetized, paralyzed primates have been reported in detail elsewhere (Carandini et al., 1997; O'Keefe and Movshon, 1998). Briefly, animals were premedicated with atropine sulfate $(0.05 \mathrm{mg} / \mathrm{kg})$ and diazepam (Valium, $1.5 \mathrm{mg} / \mathrm{kg}$ ) $30 \mathrm{~min}$ before anesthesia was induced with ketamine $\mathrm{HCl}(10.0 \mathrm{mg} / \mathrm{kg})$. We continued anesthesia on $3 \%$ isoflurane in a $98 \% \mathrm{O}_{2} / 2 \% \mathrm{CO}_{2}$ mixture during the initial surgery. We inserted catheters into the saphenous veins of the hindlimbs and performed a tracheotomy. We mounted the animal in a stereotaxic apparatus and made a craniotomy and durotomy over the opercular portion of V1 and then discontinued gas anesthesia. Anesthesia was maintained throughout the experiment by a continuous infusion of sufentanil citrate (typically 4 $\mu \mathrm{g} / \mathrm{kg}$, established for each animal) mixed with a lactated Ringer's solution (Normosol). Infusion solutions were mixed to $2.5 \%$ dextrose concentration to provide adequate nutrition, and infusion rate was adjusted to maintain fluid balance $\left(\sim 4-8 \mathrm{ml} \cdot \mathrm{kg}^{-1} \cdot \mathrm{hr}^{-1}\right)$. Vital signs (EEG, ECG, end-tidal $\mathrm{P}_{\mathrm{CO}}$, temperature, and lung pressure) were monitored continuously. Expired $\mathrm{P}_{\mathrm{CO}_{2}}$ was maintained between 3.8 and $4.0 \%$. Rectal temperature was maintained near $37^{\circ} \mathrm{C}$ through the use of a heating pad. To minimize eye movements, the animal was paralyzed with a continuous intravenous infusion of vecuronium bromide (Norcuron, $0.1 \mathrm{mg} \cdot \mathrm{kg}^{-1} \cdot \mathrm{hr}^{-1}$ ). The pupils were dilated with topical atropine, and the corneas were protected with gas-permeable hard contact lenses. We used supplementary lenses to bring the retinal image into focus by direct ophthalmoscopy. We later adjusted the refraction further to optimize the response of recorded units. We gave daily injections of a broad-spectrum antibiotic (Bicillin) and an anti-inflammatory agent (dexamethasone). Experiments typically lasted 4-5 d. All procedures complied with guidelines approved by the New York University Animal Welfare Committee.

In most experiments, we recorded with tungsten-in-glass microelectrodes (Merrill and Ainsworth, 1972) that were advanced with a hydraulic microdrive through a small durotomy made within a craniotomy of $\sim 10 \mathrm{~mm}$ diameter. In a few of the experiments, we recorded with quartz-platinum-tungsten microelectrodes (Thomas Recording, Giessen, Germany) advanced with a mechanical microdrive system. The craniotomy was typically centered $4 \mathrm{~mm}$ posterior to the lunate sulcus and $10 \mathrm{~mm}$ lateral to the midline. We recorded V1 neurons both on the operculum and in the calcarine sulcus, where the receptive field eccentricities are typically $2-5$ and $8-25^{\circ}$ of visual angle, respectively. All of our receptive fields were within $25^{\circ}$ of the fovea, and most were within $10^{\circ}$. Signals from the microelectrode were amplified and bandpass filtered, and we isolated single units with a dual-window time-amplitude discriminator (Bak, Germantown, MD). The time of each action potential was recorded with a resolution of $0.25 \mathrm{msec}$ by a CED-1401 Plus laboratory interface (Cambridge Electronic Design, Cambridge, UK).

Visual stimulus generation. We displayed all visual stimuli at a resolution of $1024 \times 731$ pixels and a video frame rate of $100 \mathrm{~Hz}$ on either a Nanao T550i or an Eizo T550 monitor. We used look-up tables to correct for nonlinearities in the relation between input voltage and phosphorluminance in the monitors. We generated drifting sinusoidal grating stimuli with a Cambridge Research Systems VSG 2/2 board (Kent, UK) running on an Intel $\times 86$-based host computer and random dot stimuli with a Silicon Graphics workstation. The mean luminance of the display was $\sim 33 \mathrm{~cd} / \mathrm{m}^{2}$ when gratings were displayed. All of the gratings were presented at $100 \%$ contrast in a circular aperture surrounded by a gray field of the average luminance.

For each isolated neuron, we began by mapping its receptive field for each eye on a tangent screen by hand. We determined the dominant eye to be that which yielded the larger response and occluded the other eye. Using a front surface mirror, we brought the receptive field into register with the center of the video monitor placed between 80 and $180 \mathrm{~cm}$ from the animal's eye, where it subtended between 10 and $22^{\circ}$ of visual angle. We then proceeded with experiments under computer control.

Experiments consisted of multiple blocks of stimuli, each composed of a randomly ordered group of all the stimuli in a set. All stimuli within a block were equal in duration and were separated by presentation of a uniform blank background (mean gray for sinusoidal stimuli and mean gray or black for Glass pattern stimuli, depending on the experiment) for $\sim 1.5 \mathrm{sec}$.

We characterized the response properties of the cell to gratings in this
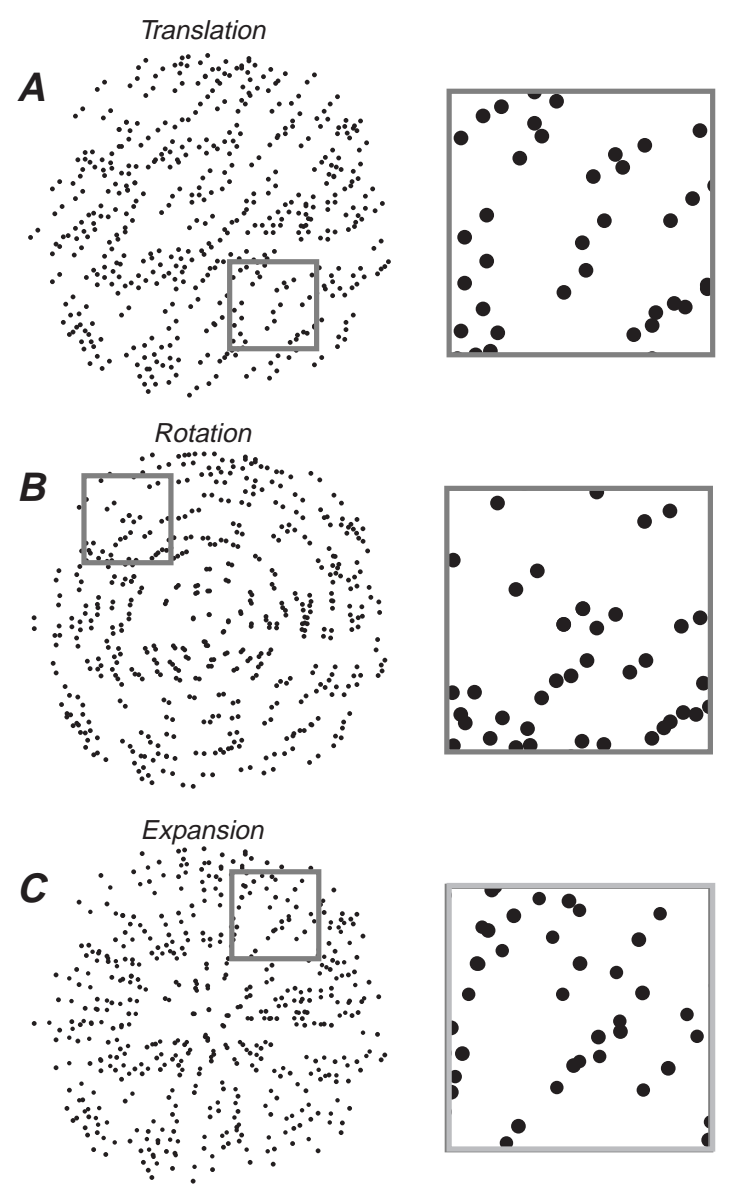

Figure 1. Examples of Glass pattern stimuli. $A$, A translational Glass pattern consists of a field of random dots shifted by a distance $r$ in a direction $\theta$ and added to itself. $B$, A concentric, or rotational, Glass pattern is created by rotating a field of random dots about the center. $C$, A radial, or expansion, Glass pattern, is obtained by multiplying the radial component of each dot by a constant. The square apertures indicate hypothetical receptive fields of V1 neurons that would contain only a portion of the stimulus. The region within the aperture for the rotational $(B)$ and radial $(C)$ patterns can be approximated by a translational pattern like that in $A$.

order: (1) orientation and direction tuning; (2) spatial frequency tuning; (3) temporal frequency tuning; and (4) size tuning. We chose a small patch of optimized grating and adjusted the vertical and horizontal position by hand to obtain the maximal response. This patch was taken to be centered in the receptive field. We classified cells as simple or complex using the standard F1/DC ratio (Movshon et al., 1978a; Skottun et al., 1991), where DC is the mean firing rate (minus baseline) and F1 is the amplitude of the Fourier component at the fundamental frequency of the response to an optimized drifting grating. Units were classified as simple if the F1/DC ratio of their spatial frequency tuning curves was $>1$, whereas all other units were classified as complex.

Glass pattern characterization. Glass pattern stimuli consisted of randomly positioned dot pairs in which dot separation and pair orientation were constant across all pairs on a given trial (Fig. 1). On each video frame (every $10 \mathrm{msec}$ ), a new set of dot pairs was plotted that was independent of the previous frame. Thus, these patterns had local spatial structure within frames but no coherent spatial structure or motion between frames. We used these dynamic patterns to randomize the positions of the dots in the pattern over time and to minimize retinal adaptation at particular dot positions. All dot patterns were presented within a circular aperture.

All Glass pattern stimuli were presented for $1 \mathrm{sec}$, immediately preceded and followed by $500 \mathrm{msec}$ periods of dynamic random dots (with the same mean luminance on each video frame). This allowed us to avoid contamination of the Glass pattern response by any response to the 
luminance change caused by the onset of the dots. An additional $2 \mathrm{sec}$ stimulus of purely random dots and a blank screen were included in each block of trials, from which we measured baseline responses.

Dots were usually presented at maximum contrast (i.e., white dots on a black background). The maximum luminance was $68.4 \mathrm{~cd} / \mathrm{m}^{2}$, and the minimum was near $0.0 \mathrm{~cd} / \mathrm{m}^{2}$. The mean luminance of the display was $\sim 0.2 \mathrm{~cd} / \mathrm{m}^{2}$ when white dots were displayed on a black background. In some experiments, we presented maximum luminance ("white") or minimum luminance ("black") dots on a mid-gray background $\left(34.2 \mathrm{~cd} / \mathrm{m}^{2}\right)$. All of these stimuli, in which the dots all have the same luminance, are called same-polarity patterns. We also used opposite-polarity patterns, in which half of the dots were white, the other half were black, and the background was mid-gray. For opposite-polarity Glass patterns, each pair consisted of one white and one black dot. The white dot in each pair was chosen at random. Dot size was typically $0.04^{\circ}$ (range, $0.03^{\circ}-0.12^{\circ}$ ) and density was typically 200 dots per degrees squared per second (range, $100-800)$. In this range, human observers readily perceive Glass patterns, and variations in dot density have no significant impact on perception (Alliston et al., 2001).

For each neuron, we presented a matrix of Glass patterns with eight orientations $(\theta)$ and five dot separations $(r)$. The orientations were evenly spaced over $180^{\circ}$. We chose the range of $r$ to include values from approximately $\lambda / 4$ to $3 \lambda / 2$, where $\lambda$ was the preferred spatial period of the cell (see below). In the opposite-polarity and size experiments, we used the value of $r$ determined to optimize the response of the cell. For the size experiments, we chose the orientation to be aligned to the optimal orientation for gratings.

Quantitative measures. We collected responses for gratings, Glass patterns, and random dots as a function of stimulus size. We fit data from these size tuning curves with the integral of a difference of Gaussians (DeAngelis et al., 1994). We chose the size of the classical receptive field (CRF) to be the smallest diameter grating for which the fitted curve reached $95 \%$ of its maximum. Optimal sizes for Glass pattern and random dot stimuli were chosen in the same manner. We also fit descriptive functions to spatial frequency tuning curves for gratings to find the optimal spatial period, $\lambda$ (the inverse of the optimal spatial frequency), for each cell (Levitt et al., 1994).

To characterize orientation tuning curves, we determined the selectivity and preferred angle by calculating a tuning bias vector (Leventhal et al., 1995; O'Keefe and Movshon, 1998), similar to the vector strength calculation introduced by Levick and Thibos (1982). We represented an orientation tuning curve as a set of vectors, $\left(\theta_{\mathrm{n}}, R_{\mathrm{n}}\right)$, where $\theta_{\mathrm{n}}$ is stimulus orientation, $R_{\mathrm{n}}$ is the response magnitude (with baseline subtracted), and $n$ is an index from 1 to the number of points, $N$, in the tuning curve. The preferred orientation is given by the circular mean angle:

$$
\frac{1}{2} \arctan \left(\frac{\sum_{n=1}^{N} R_{n} \sin \left(2 \theta_{n}\right)}{\sum_{n=1}^{N} R_{n} \cos \left(2 \theta_{n}\right)}\right) .
$$

To measure selectivity, we calculated the summed response vector:

$$
v=\sum_{n=1}^{N} R_{n} e^{\left(i 2 \theta_{n}\right)},
$$

and normalized its magnitude by the summed magnitude of all the response vectors:

$$
\text { selectivity index }=\frac{|v|}{\sum_{n=1}^{N} R_{n}\left|R_{n}\right|} .
$$

The selectivity index is 0 for a cell responding equally at all orientations and 1 for a cell that responds only to a single orientation. To estimate the significance of each selectivity estimate, we used the permutation technique described in O'Keefe and Movshon (1998). For each tuning curve, we performed the selectivity index analysis on 2000 random permutations of the data and considered a measured selectivity index to be significant if it exceeded the 90th percentile of the permuted distribution.
To estimate analogous quantities for tuning curves with four lobes (rather than two), which we term "quadropoles," we modified the first two equations simply by substituting $4 \theta_{\mathrm{n}}$ for $2 \theta_{\mathrm{n}}$ and taking one-quarter rather than one-half of the arctangent. This results in a measure of preference and bias appropriate for functions with periodic peaks and troughs every $90^{\circ}$, rather than every $180^{\circ}$.

\section{RESULTS}

We made extracellular recordings from 113 neurons (38 simple, 75 complex) in the primary visual cortex of 13 macaque monkeys. We characterized each cell with drifting sine wave gratings before testing with dynamic, translational Glass patterns. We did not test neurons that were not selective for the orientation of gratings, but did not otherwise exclude neurons from study with Glass patterns. Not all of the experiments described herein were performed on every cell. In our population of cells, the distribution of orientation bandwidths $\left(\right.$ mean $\left.=64.5^{\circ}, \mathrm{SD}=25.9^{\circ}\right)$ and spatial frequency peak $($ mean $=2.1 \mathrm{c} / \mathrm{deg}, \mathrm{SD}=1.2 \mathrm{c} / \mathrm{deg})$ and bandwidth $($ mean $=$ 2.2 octaves, $\mathrm{SD}=0.7$ ) were similar to those found by other investigators at the eccentricities of our recorded cells (DeValois et al., 1982; Foster et al., 1985) and to the larger population of neurons recorded in our laboratory for other experiments.

To provide a framework for interpreting the neuronal responses to Glass patterns, we begin by describing theoretical responses of oriented filters, designed to represent V1 receptive fields, to these stimuli. The intuition gained from this exercise guides our data analysis.

\section{Tuning of oriented filters to Glass patterns}

Figure $2 A$ shows a linear spatial filter that represents the spatial receptive field of a V1 simple cell as a Gabor function (Marčelja, 1980). We chose the parameters to match the shape, orientation, and spatial frequency selectivity of typical simple cells in monkey or cat V1 (DeValois et al., 1982, 1985; Foster et al., 1985; Jones and Palmer, 1987). Light and dark shading represent sensitivity to light increments and decrements, respectively. The essential aspects of the tuning of such a filter to Glass patterns as a function of orientation and dot separation can be grasped by considering the alignment of a single pair of dots with the receptive field. First, consider the case where the dot separation, $r$, is half of the optimal spatial period, $\lambda$ (Fig. $2 A$ ). When such a dot pair is orthogonal to the receptive field, the signals elicited by the dots will tend to cancel. For example, the unmarked dot and the dot marked "-" fall in opposite-signed regions of the receptive field (Fig. $2 A$ ). When the pair is aligned to the receptive field, however, the dots will tend to reinforce because they fall in samesigned regions (Fig. $2 A$, plain and + dots). The cancellation and reinforcement as a function of $\theta$ determine the orientation tuning curve for the filter [(Fig. $2 C$, polar plot) taken from Fig. $9 B$ where we derive the response for all values of $r$ and $\theta$ ]. Now, consider the case of $r=\lambda$, where the dot separation matches the spatial period of the receptive field (Fig. $2 B$ ). The parallel alignment of the dot pair again causes response reinforcement, but the orthogonal alignment now escapes cancellation because one dot falls beyond the inhibitory flank of the filter. Around $30^{\circ}$ from parallel, there is response cancellation. Thus, for $r=\lambda$, the model predicts the four-lobed tuning curve shown in Figure $2 D$. If the sign of one dot in each pair is inverted so that the dots are of opposite contrast, the orientation tuning curves will be inverted (Fig. $2 E, F$ ) because dot pairs that previously canceled will now reinforce, and vice versa. In the Appendix we provide an alternate approach to visualizing the tuning of a Gabor function to Glass patterns by examining the Fourier representation of both the filter and stimulus in 
$\boldsymbol{A}$

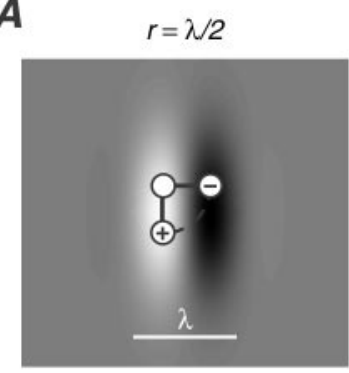

B

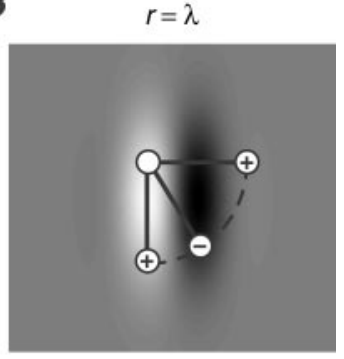

C

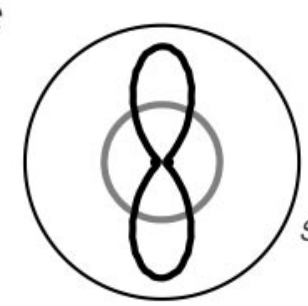

D

Same polarity dot pairs

E

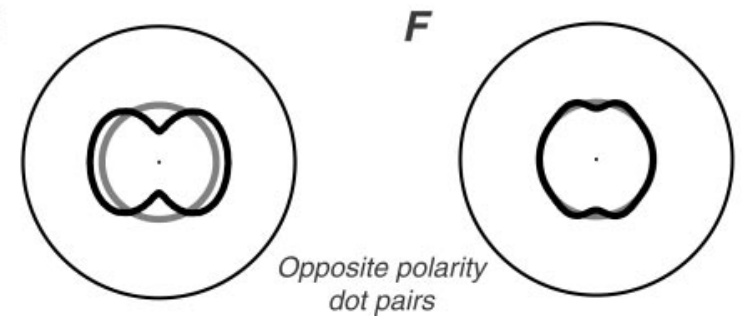

Figure 2. Modeling the response of a V1 cell to Glass patterns. $A$, The spatial profile of a V1 simple cell receptive field is modeled as a Gabor function (a Gaussian $\times$ a sinusoid) with an aspect ratio of 0.6 , frequency of $2.16 \mathrm{c} / \mathrm{deg}$, and width (1 SD of Gaussian) of $0.16^{\circ}$. These are typical values for a macaque V1 simple cell (DeValois et al., 1982; Foster et al., 1985; Parker and Hawken, 1988) and are also representative of simple cells in cat area 17, which have similar structure (DeValois et al., 1985). Black represents negative values, white represents positive, and background gray is zero. Pairs of dots with separation, $r$, equal to half the spatial period, $\lambda$, of the grating are superimposed on the receptive field at several angles. Responses to dots in a pair aligned parallel to the grating reinforce $(+)$, whereas responses to dots in a pair orthogonal to the grating cancel $(-)$. $B$, For $r=\lambda$, however, both parallel and orthogonal alignments reinforce $(+)$, whereas some intermediate angles cancel $(-)$. $C, D$, Orientation tuning curves (response vs $\theta$ ) are plotted in polar coordinates for the $r$ values in $A$ and $B$. For $r=\lambda / 2$ the tuning is bi-lobed, similar to the classical tuning to edges and sine waves. For $r=\lambda$, the tuning becomes four-lobed. Gray circles represent responses to random dots. $E, F$, Orientation tuning curves similar to $A$ and $B$, but for oppositepolarity (i.e., one white and one black dot). In $E$, the tuning is shifted by $90^{\circ}$ and is wider for $r=\lambda / 2$. In $F$, for $r=\lambda$, the selectivity is nearly abolished. Gray circles represent responses to opposite-polarity random dots (half black and half white).

the frequency domain, where Glass patterns have a convenient representation (DeValois and Switkes, 1980; Dakin, 1997b).

We have so far considered only responses to a pair of dots at a particular location in the receptive field, but dot pairs are placed randomly in Glass patterns. Local responses to randomly positioned dot pairs will tend to cancel for a purely linear filter like that in Figure 2 because dots are as likely to fall in the inhibitory region as in the excitatory region. Cortical cells, however, produce rectified responses, which we simulated by applying a threshold to the model output (Movshon et al., 1978a). Such a rectified output provides a signal related to the variance of the combined local activations. The tuning curves (Fig. $2 C-F$ ) are based on rectified responses averaged across all possible positions of a particular dot pair; the baseline for the simulated tuning curves (gray circles) is the response of the model to randomly placed dots of the same density as the Glass patterns. Our simulations were specifically designed to predict the responses of simple cells, but they also capture the responses of complex cells that sum the rectified outputs of linear filter subunits (Movshon et al., 1978b) and of even- and odd-symmetric filters (see Appendix).

The model makes several interesting predictions. (1) The shape of the orientation tuning curve for Glass patterns should depend on $r / \lambda$, the ratio of the dot separation to the preferred spatial period of the cell. (2) The response to an optimally oriented Glass pattern will exceed that to random dots, whereas the response to the least effective orientation will fall below that baseline. (3) For typical receptive fields, the greatest degree of orientation selectivity should occur for $r$ between $\lambda / 4$ and $\lambda / 2$, and the maximal response should occur when the dot-pair orientation matches the classical preferred orientation of the cell. (4) A second mode of orientation tuning with four principal lobes is possible for relatively large dot separations; as detailed in the Appendix, the strength of this mode depends on the specific properties of the linear filter used. (5) For Glass patterns made with oppositepolarity (black-white) dot pairs, the tuning is inverted compared with that of a same-polarity pattern. In particular, the preferred orientation will rotate to be orthogonal to the receptive field orientation, and orientation tuning will be broader and less modulated than for a same-polarity pattern of the same $r$. We will now consider how well these predictions hold for the responses of V1 neurons.

\section{Orientation tuning of V1 cells to Glass patterns}

Figure 3 shows the orientation tuning curves of a V1 cell plotted in polar coordinates. For grating stimuli, $180^{\circ}$ opposite points indicate opposite directions of drift for the same orientation. However, for Glass pattern stimuli, which have no coherent motion, we reflected the orientation data to the $180^{\circ}$ opposite point (which has the same orientation). For two example cells, Figure 3, $A$ and $B$, we show tuning for the direction of a drifting sinusoidal grating (dotted lines). The Glass pattern orientation tuning curve for $r \approx \lambda / 2$ is overlaid (thick lines) on those curves, with the neuronal response to random dots taken as the baseline (light gray circle). The peaks of the orientation tuning curves match, which indicates that with a dot separation of $r \approx \lambda / 2$, both cells preferred a Glass pattern in which the dot pairs were parallel to the bars of the preferred drifting grating. In both cases, the response to the preferred Glass pattern was substantially smaller than the response to the preferred grating. Figure 3, $C$ and $D$, shows, at an enlarged scale, the response of the same two cells to Glass patterns when $r \approx \lambda / 2$ and $r \approx \lambda$. When $r \approx \lambda / 2$, the tuning curves have two lobes, whereas four-lobed tuning is discernible when $r \approx \lambda$.

We collected orientation tuning curves for Glass patterns at multiple values of $r$. The distribution of the values of $r / \lambda$ for which the tuning curve showed the highest orientation selectivity index (see Materials and Methods) is shown in Figure $4 A$. For most cells, the highest selectivity index occurred for a value of $r / \lambda$ below 0.6 , and the optimal values for $r$ and $\lambda$ were highly correlated (Pearson's $r=0.66 ; p<0.0001$ ). This was consistent with predictions from our model (see Appendix). For each cell, we chose the orientation tuning curve with the strongest response modulation where $r / \lambda<0.6(r \approx \lambda / 2$, range $0.2-0.6)$, and where $r$ was as close to $\lambda(r \approx \lambda$, range $0.7-1.3)$ as possible. We estimated the optimal orientation and the degree of orientation selectivity from a vector strength calculation (see Materials and Methods). 
$\boldsymbol{A}$

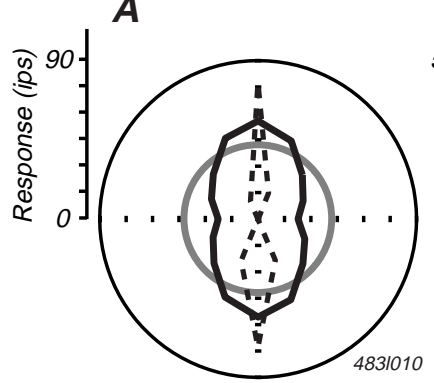

$B$
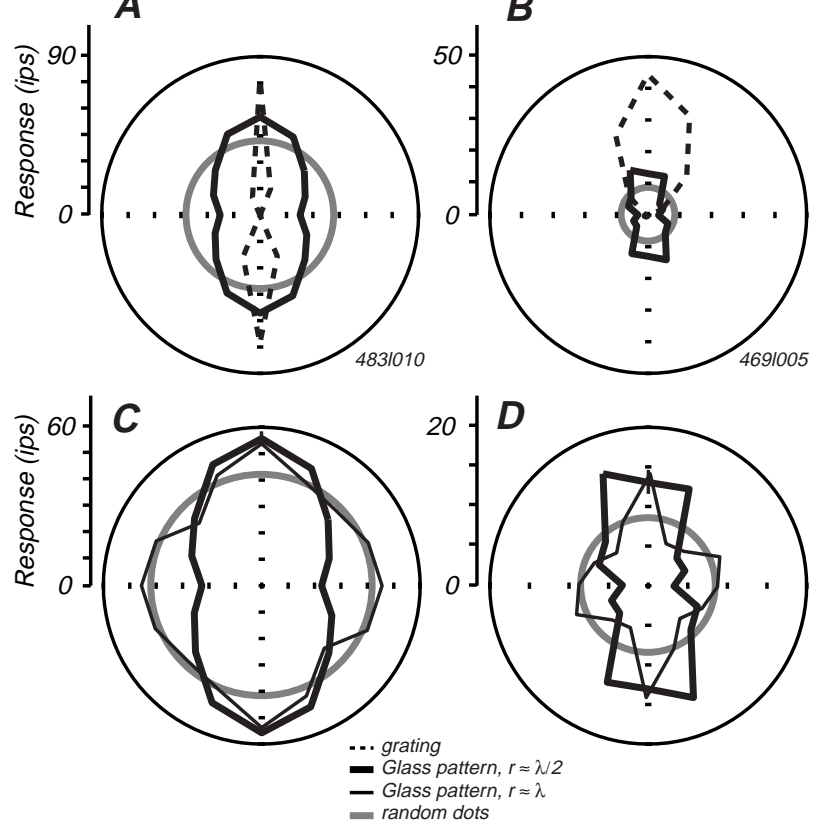

Figure 3. A comparison of orientation tuning for gratings and Glass patterns for two example cells. $A$, Polar plots of orientation tuning curves are shown for a simple cell responding to drifting sinusoidal gratings (dotted line $)$ and Glass patterns $(r \approx \lambda / 2)$ (thick black line). All curves were rotated so that the peak of the grating tuning curve points upward. The preferred orientation for Glass patterns matches that for gratings. $C$, The Glass pattern tuning curve from $A$ is replotted at an enlarged scale along with the Glass pattern tuning curve for $r \approx \lambda$ (thin black line). The shapes of these orientation tuning curves were consistent with predictions from our model (Fig. 2). The thicker curve shows greater selectivity, whereas the thinner curve is roughly four-lobed. The same trends are evident in the plots for a complex cell shown in $B$ and $D$ (same format as $A$ and $C$ ). Gray circles show responses to random dots with the same dot density as the Glass pattern. ips, Impulses per second.

In Figure 4, $B$ and $C$, we show the distributions of differences in optimal orientations for Glass patterns relative to gratings when $r \approx \lambda / 2$ and $r \approx \lambda$, respectively. When $r \approx \lambda / 2$ (Fig. $4 B$ ), $>70 \%$ of neurons showed an orientation preference for Glass patterns that was within $\pm 22.5^{\circ}$ of that to gratings. When $r \approx \lambda$ (Fig. $4 C$ ), the trend was similar, but more neurons had disparate preferences for Glass patterns and gratings. This was because of decreased modulation and selectivity in the tuning curves at larger dot separations.

To compare the shape as well as the peak of orientation tuning for Glass patterns across our population of cells, we scaled each tuning curve to have a maximum value of 1 . The averages of all such normalized curves for $r \approx \lambda / 2$ (bold curve) and $r \approx \lambda$ (thin curve) are shown in Figure $4 D$. These mean tuning curves resemble those predicted by our simulations shown in Figure 2; note in particular that there is a suggestion of a four-lobed structure, as predicted in Figure $2 D$, for $r \approx \lambda$.

To further explore the prevalence of four-lobed orientation tuning and its relationship to $r$, we modified the orientation selectivity index (see Materials and Methods). The usual index is designed to be sensitive to peaks and troughs differing by $180^{\circ}$, so we performed an analogous calculation sensitive to peaks and troughs differing by $90^{\circ}$ to find values for the optimal "quadropole angle" and "quadropole selectivity" for $r \approx \lambda / 2$ and $r \approx \lambda$. The optimal quadropole angles, as expected, were well aligned with the optimal orientations when the quadropole tuning was robust.
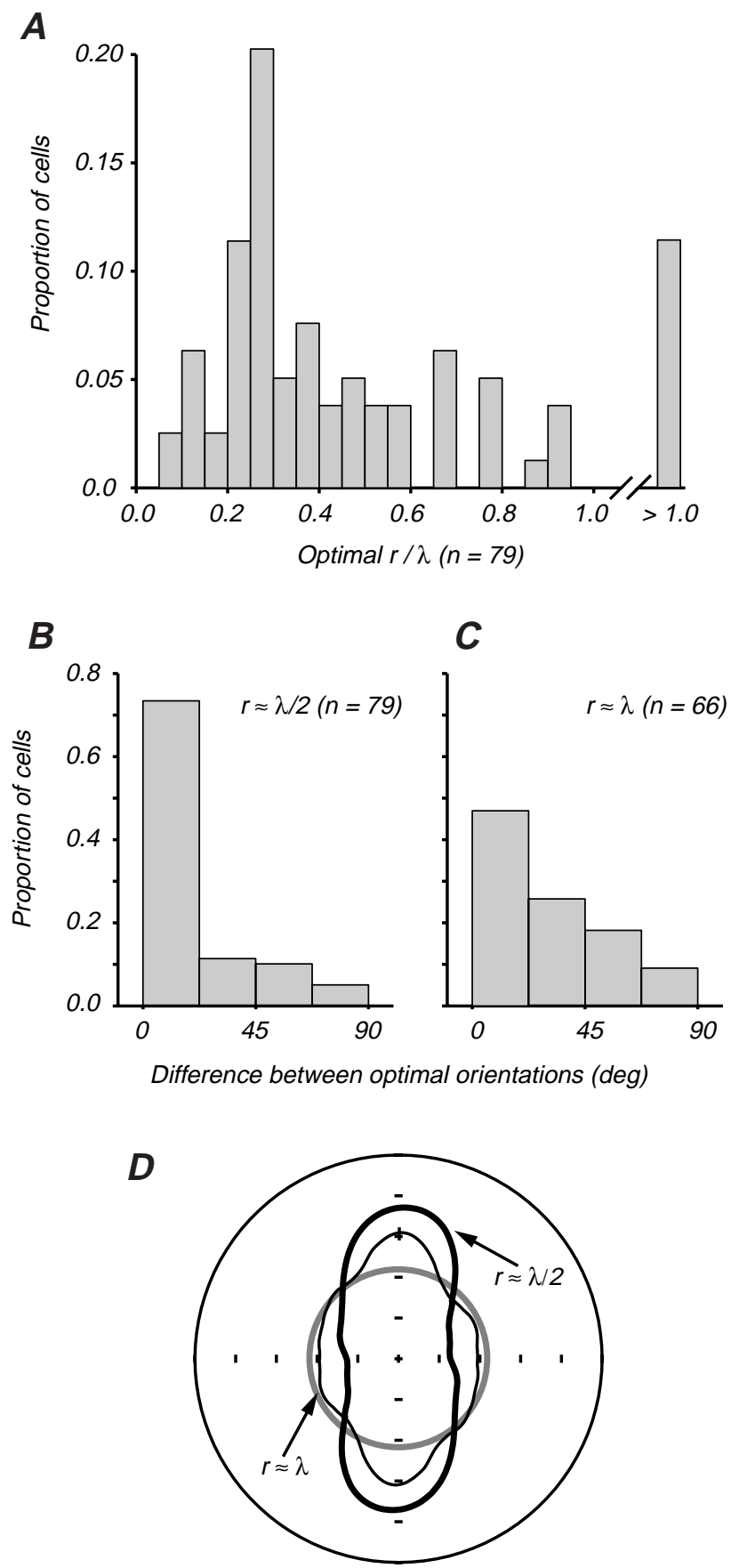

Figure 4. Preferred Glass pattern configuration across the population. $A$, The frequency histogram of $r / \lambda$ values associated with the maximal selectivity in the Glass pattern tuning curves shows that selectivity was highest for most cells (72\%) when dot separation was $<0.6$ of the optimal spatial frequency. $B$, The frequency histogram of the absolute value of the difference between the preferred grating orientation and the preferred Glass pattern orientation at $r / \lambda<0.6$ has a prominent peak near $0^{\circ}$. Thus, Glass pattern tuning was predictable from grating tuning. Simple and complex cells had similar distributions and are combined here. $C$, For $r \approx$ $\lambda$, the trend in $B$ is weaker but still present. $D$, The average of all tuning curves, normalized to have a minimum response of zero and a maximum response of 1 before averaging, shows a result consistent with the histograms in $A$ and $B$. The bold curve $(r \approx \lambda / 2)$ is bi-lobed, and the thin curve $(r \approx \lambda)$ has a roughly four-lobed structure, as predicted. An error bar $( \pm 1$ SEM) is shown at the peak of the $r \approx \lambda$ tuning curve. The gray circle shows the normalized response to random dots. 
Table 1. Selectivity of orientation tuning for Glass patterns

\begin{tabular}{lllllll}
$r$ & \multicolumn{2}{l}{ Selectivity index } & & & \multicolumn{2}{l}{ Fraction significant } \\
\cline { 2 - 3 } \cline { 6 - 7 } & Orientation & Quadropole & & & Orientation & Quadropole \\
\hline$\lambda / 2$ & 0.47 & 0.27 & 0.0001 & $37 / 79$ & $4 / 79$ & 0.0001 \\
$\lambda$ & 0.33 & 0.35 & 0.701 & $8 / 66$ & $15 / 66$ & 0.169 \\
& 0.0002 & 0.013 & Significance & 0.0001 & 0.0038
\end{tabular}

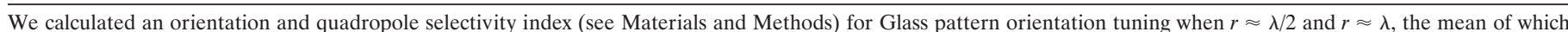

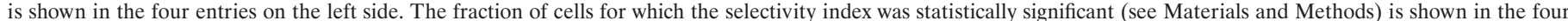

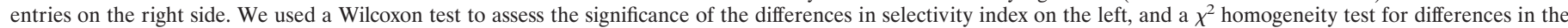

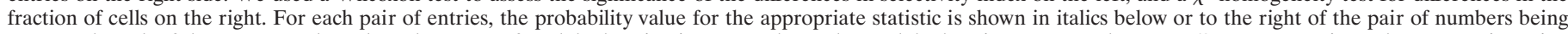

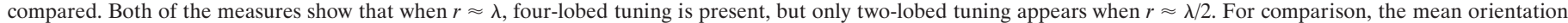
selectivity index for gratings was 0.61 , with 78 of 79 tuning curves significantly selective.

Table 1 shows the magnitude and prevalence of orientation and quadropole selectivity. The left part of Table 1 shows means of the selectivity index for both orientation tuning and quadropole tuning; the values in italics give the significance of the difference between the adjacent values. The mean selectivity for orientation was largest when $r \approx \lambda / 2$, whereas the mean quadropole selectivity index was largest when $r \approx \lambda$. We also tested the significance of the selectivity index of each cell against randomly permuted data (see Materials and Methods). The right part of Table 1 shows the fraction of cells that showed significant selectivity, with the values in italics again representing significance values. The fraction of orientation-selective cells was significantly higher when $r \approx \lambda / 2$, and the fraction of quadropole selective cells was higher when $r \approx \lambda$.

Our model predicts that cells with narrow tuning for orientation and spatial frequency should be the most likely to show four-lobed tuning (see Appendix). We used these two measures of the selectivity of the response of a cell to gratings and correlated them with the quadropole selectivity index measured when $r \approx \lambda$. Orientation bandwidth at half-height in degrees (Pearson's $r=-0.18 ; p=0.155)$ and spatial frequency bandwidth in octaves (Pearson's $r=-0.19 ; p=0.127$ ) show correlations in the expected directions, although they are not statistically significant.

In summary, significant four-lobed tuning was present in roughly one-quarter of our cells and was weakly correlated with tuning bandwidth. As we show in the Appendix, the expected strength of four-lobed tuning is relatively modest, even for narrowly tuned cells. We would therefore expect four-lobed tuning to be prominent only in a fraction of V1 cells. The reliability of this effect across the population is confirmed by the average tuning curve for $r \approx \lambda$ (Fig. 4D), which shows a four-lobed structure that is in the expected orientation relative to Glass pattern tuning when $r \approx \lambda / 2$. We thus confirm the model prediction that conventional two-lobed orientation selectivity should dominate tuning curves measured for $r \approx \lambda / 2$ and that four-lobed selectivity should be evident for some cells when $r \approx \lambda$.

\section{Response strength}

V1 cells showed clear preferences for Glass pattern orientation, but their responses to these patterns were generally much weaker than to drifting gratings. Because Glass pattern tuning involves modulation about a mean rate of response to random dots, we took the difference between the maximum and minimum responses in a Glass pattern tuning curve as the modulation of response. We compared this with the modulation of response in the grating tuning curve. For each cell, Figure 5 plots the modulation of response (peak-trough) to Glass patterns against that to gratings. All points fell well below the unity line, indicating that

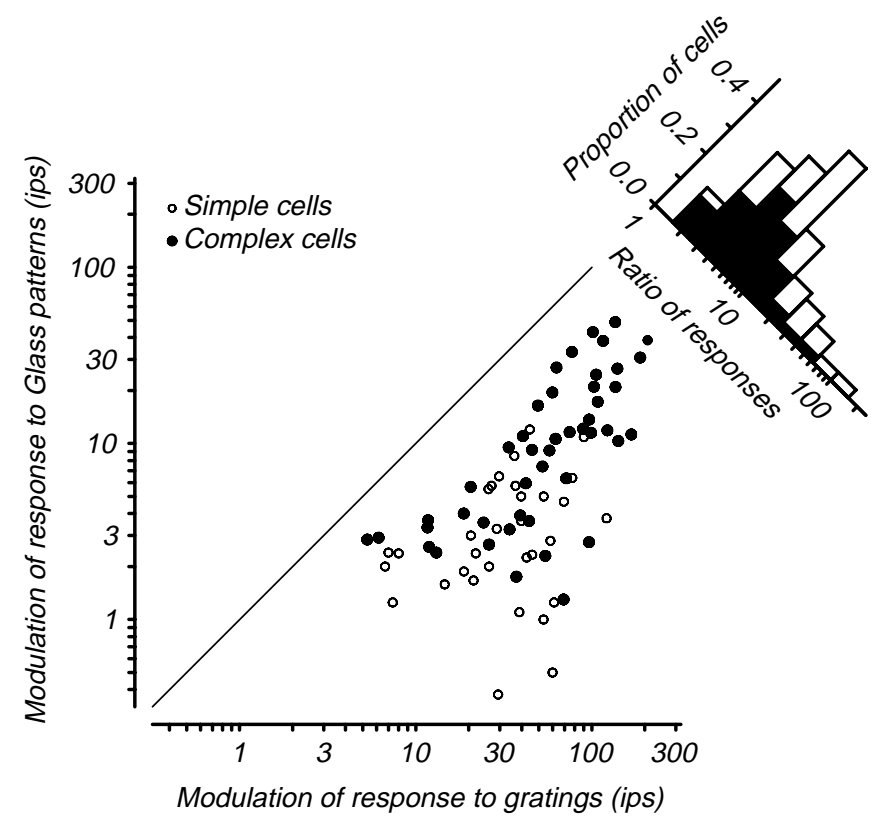

Figure 5. Responses to Glass patterns were weaker than those to gratings. For both complex cells (filled circles) and simple cells (open circles), we defined the response modulation to be the peak minus the trough of the orientation tuning curve. For Glass patterns, we used the orientation tuning curve taken at the optimal dot separation. All points fall below the diagonal line of equality, indicating that all cells responded better, i.e., had more modulated tuning curves, to gratings than to Glass patterns. The response ratio (oblique histograms) was $\sim 10$ on average. The absolute peak firing rates for Glass patterns were also much lower than those for gratings (complex cells, mean $=28.5$ spikes/sec; simple cells, mean $=8.6$ spikes/sec).

the neurons responded more vigorously, often by more than an order of magnitude, to gratings (complex cells, geometric mean $=$ 52.1 vs 8.6 spikes/sec; simple cells, geometric mean $=28.1$ vs 2.8 spikes/sec). The marginal distribution of the ratios of responses for each cell is shown in the oblique histograms at the top right. The geometric mean of the response ratio of gratings to Glass patterns was 10.1 for simple cells and 6.0 for complex cells.

From a linear spatial filter we can predict not only the orientation tuning to Glass patterns but also the response ratio. The ratio in response that we observed was roughly half as large as our simulations predicted for a range of dot size and density values that spanned the range used experimentally. Thus the cells we studied responded more strongly to Glass patterns than the linear model predicts. This could be caused by response saturation to the high-contrast grating stimuli or because the cells operate in a 
state of high gain when confronted with the dot stimuli, which have low-contrast energy in any given spatial frequency band (Carandini et al., 1997).

\section{Opposite-polarity dot pairs}

A feature of Glass pattern perception that suggests a limitation of local orientation sensors is the relative weakness of percepts elicited by patterns in which the two members of a dot pair are of opposite contrast polarity, i.e., one black and one white dot on a gray background (Glass and Switkes, 1976; Prazdny, 1986; Kovács and Julesz, 1992; Dakin, 1997b; Wilson et al., 1997). Figure 6, A and $B$, shows a same-polarity and opposite-polarity translational Glass pattern made from the same set of seed dots. Because the dot pairs in both stimuli have the same orientation, in principle a feature-detection system could respond to both patterns in a similar manner. However, if neuronal responses approximate the output of linear spatial filters, they would give sharply different tuning curves to same- and opposite-polarity dot pairs (Fig. 2, compare $C, E)$. Recall that our model predicts that oppositepolarity Glass pattern orientation tuning curves should be orthogonal to regular Glass pattern curves and that selectivity and modulation strength should be poorer for the opposite contrast patterns (Fig. $2 E, F$ ). In addition, the optimal dot separation predicted by the model is the same for same- and opposite-polarity dot pairs. We tested this prediction by comparing the tuning for same- and opposite-polarity dots for the optimal dot separation. Figure 6, $C$ and $D$, shows this comparison for two cells. In each case, inverting the contrast polarity of the patterns shifted the tuning curves by $\sim 90^{\circ}$, drastically reduced the response magnitude, and reduced the degree of orientation selectivity.

Our same-polarity patterns consisted of white dots on a black background. Opposite-polarity patterns must have a gray background, so the contrast in the stimulus was inevitably reduced. Because of the decreased contrast present, we expected responses to be weaker to opposite-polarity patterns than those we collected with same-polarity patterns, and in fact this was the case. Many cells did not respond at all to these patterns when we used small dots $\left(0.04^{\circ}\right)$, so we increased the dot size by a factor of $2-4$ to increase responses. In some cells, we compared the responses to opposite-polarity patterns with those to same-polarity patterns with all white dots or all black dots on a mean gray background and found them to be similarly weak (Fig. $6 E, F$ ). Responses were approximately an order of magnitude less than those to samepolarity patterns, even with dot size increased. In addition, both of the same-polarity patterns showed tuning consistent with that for white dots on a black background (i.e., orthogonal to that for opposite-polarity patterns). Therefore, the reduction in response to opposite-polarity patterns (Fig. $6 C, D$ ) is caused by contrast reduction in the stimulus.

In Figure $6 G$ we show the distribution of differences in optimal orientations for opposite-polarity Glass patterns relative to gratings when $r \approx \lambda / 2$. Most of the cells had an optimal orientation for opposite-polarity Glass patterns that was nearly orthogonal (within $\pm 45^{\circ}$ ) to that for gratings. Comparing this result with that in Figure $4 B$, we note that the peaks of the distributions are offset by $\sim 90^{\circ}$. In addition, the distribution for same-polarity patterns (Fig. $4 B$ ) is more tightly clustered around $0^{\circ}$ than that for oppositepolarity patterns (Fig. $6 G$ ), indicating a difference in orientation selectivity. We conclude that opposite-polarity Glass patterns elicit responses in V1 that are less orientation selective than responses to conventional patterns and shifted by $90^{\circ}$.

\section{Effects of stimulus size}

In Figure 1 we showed that within a small aperture, such as that likely to be viewed by V1 cells, any extended Glass pattern can be approximated by a translational pattern. However, responses to small patches of a Glass pattern might not be representative of those to large stimuli. For example, form-sensitive cells in higher cortical areas could affect V1 through feedback connections, perhaps enhancing their response. Alternatively, large translational Glass patterns could engage the orientation-selective mechanisms that mediate surround suppression, thereby causing a reduction in response. It is well established that increasing the size of a grating or bar stimulus beyond the classical receptive field engages a nonclassical surround that suppresses the response of V1 cells (Hubel and Wiesel, 1968; Blakemore and Tobin, 1972; Nelson and Frost, 1978; Knierim and Van Essen, 1992; DeAngelis et al., 1994). In addition, there is evidence that cells may spatially sum over a larger area when tested with low contrast gratings (Sceniak et al., 1999; Cavanaugh et al., 2002), which are like Glass patterns in the weak responses that they evoke.

To test the effect of stimulus size on Glass pattern tuning, we performed two additional experiments. These data were collected in 4 of the 13 animals. In the first experiment (in 34 neurons), we collected size tuning curves to optimally configured Glass patterns, random (i.e., unpaired) dots, and optimally configured sinusoidal gratings (see Materials and Methods). Examples of the two main patterns of response that we observed are shown in Figure $7 A, B$. In Figure $7 A$, the response of the cell was suppressed by grating stimuli that were more than $\sim 1^{\circ}$ in diameter. The tuning curves for both types of dot stimuli showed no sign of suppression, i.e., response increased up to the maximum size tested. We observed this behavior in a minority of our cells. The example cell in Figure $7 B$ shows a different behavior. Here, the size tuning curves for dots showed signs of suppression for the same range of sizes that produced suppression for gratings (e.g., $>2^{\circ}$ ). At around the same size, we observed suppression for both types of dot stimuli.

For each size tuning curve, we found the best response to Glass patterns and compared it with the response to a random dot field of the same size. If a cell did not respond more to Glass patterns than to random dots by at least 1 SEM (indicating that the cell was not tuned for size), we removed it from further consideration.

We omitted from further analysis 10 of 34 cells, for which the peak response to Glass patterns was $<1$ SEM larger than the response to random dots. For the 24 remaining cells, we plotted the optimal size (Fig. 7C) and amount of suppression (Fig. 7D) for Glass patterns and gratings. In this population of cells, the mean optimal size for Glass patterns $\left(1.7^{\circ}\right)$ was larger than that for gratings $\left(1.0^{\circ}\right)$, and this difference was statistically significant $(t$ test; $p=0.004)$. Responses to Glass patterns showed significantly less suppression on average (0.14) than those to gratings $(0.28 ; t$ test; $p=0.007)$. Furthermore, Glass pattern and random dot tuning for size were highly correlated (Pearson's $r=0.71 ; p=0.0001$ ). Even as the cell showed suppression or summation for an increase in size, the firing rate difference between Glass pattern and random dot stimuli was maintained at a constant rate (Fig. $7 A, B$ ).

In the second experiment (in 43 neurons), we studied orientation selectivity for Glass pattern stimuli of two sizes, one that was confined to the CRF and one that included both the CRF and surround (as measured with gratings). The results from this experiment on two example cells (the same as in Fig. $7 A, B$ ) are shown in Figure $7 E, F$. Although the response increased with 

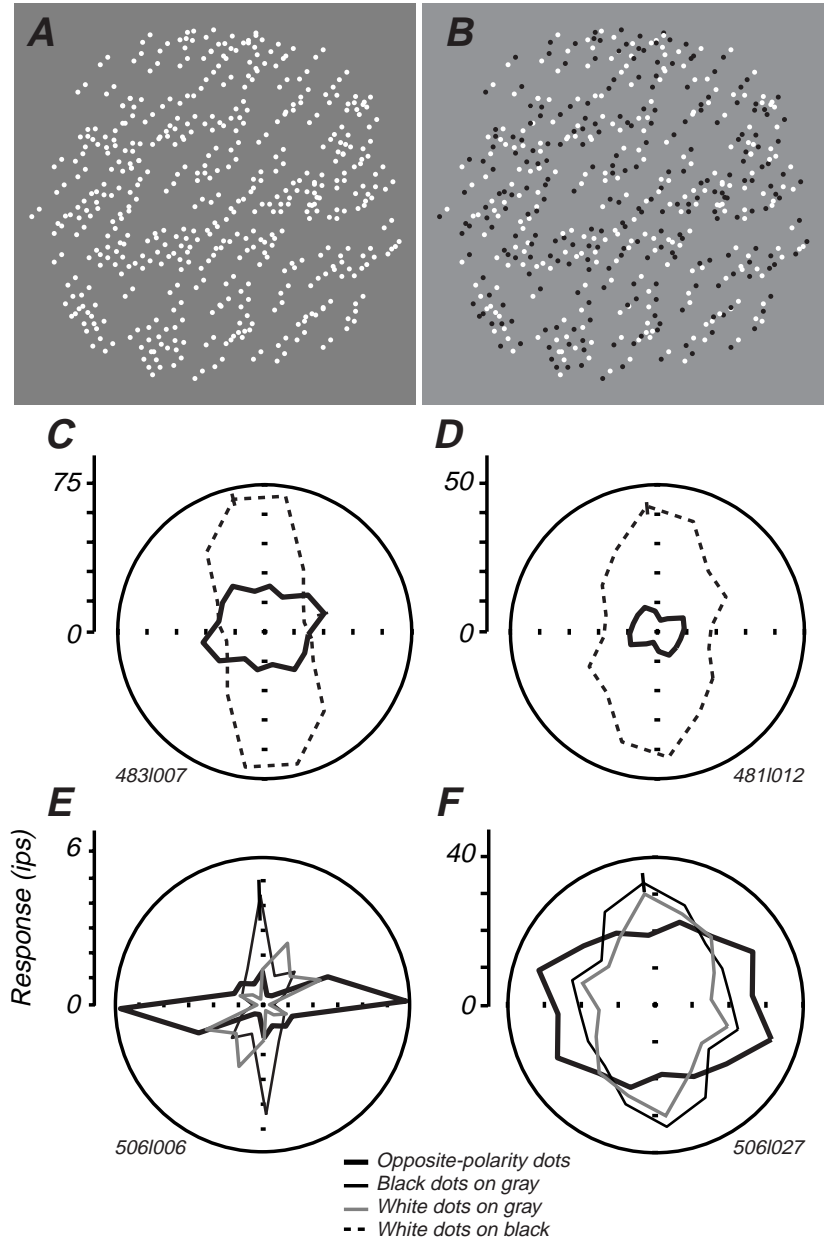

$F$

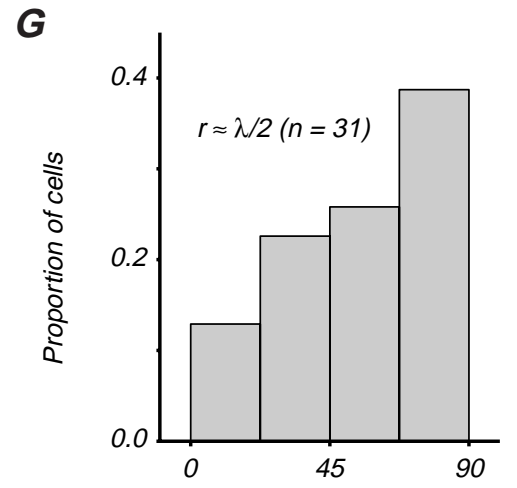

Difference between optimal orientations (deg)

Figure 6. A comparison of the responses to same- and opposite-polarity Glass patterns. $A$, The same-polarity Glass pattern shown here is displayed on a mean luminance background, as opposed to the usual black background, for comparison with opposite-polarity Glass patterns. $B$, This opposite-polarity Glass pattern has the same dot separation, orientation, and dot placement as in $A$, but it does not elicit the same percept of oriented structure. $C, D$, For two example cells, orientation tuning curves for opposite-polarity Glass patterns (thick solid line) are plotted with tuning curves to same-polarity Glass patterns displayed on a black background (dashed lines). Response amplitude for opposite-polarity patterns is less because of the reduced contrast, and there is an $\sim 90^{\circ}$ change in the orientation preference for opposite-polarity patterns. As always, orientation is plotted relative to the preferred orientation for gratings, which is plotted as upward. $E, F$, A direct comparison of orientation tuning curves for opposite- and same-polarity Glass patterns displayed on mean gray backgrounds is shown here for two example cells. Responses for same-polarity patterns ( gray lines for white dots, thin black lines for stimulus size in Figure $7 E$, the orientation preference and selectivity remained similar. In Figure $7 F$, the same is true, although the cell showed suppression with increasing stimulus size. On average across all cells, the peak firing rate for the smaller stimulus (24.8 ips) was significantly higher than for the larger pattern (21.8 ips; $t$ test; $p=0.006)$. This was also true for unpaired random dots (mean $=20.0$ ips vs $16.6 \mathrm{ips} ; t$ test; $p=0.002)$. In addition, there was no significant difference in either the optimal orientation (mean difference $=-2.89^{\circ} ; t$ test; $p=0.43$ ) or the orientation selectivity index (mean $=0.53$ vs 0.56 ; Wilcoxon test; $p=0.548$ ) in response to the two pattern sizes.

In summary, in no case did stimulation of the surround importantly modify the selectivity of the cell for Glass pattern orientation. Some cells had similar optimal sizes for Glass patterns and for gratings (Fig. 7B), whereas others had larger optimal sizes for Glass patterns because suppression for large gratings was weaker or absent for large Glass patterns (Fig. 7A). The behavior in this latter group of cells is consistent with the findings of Casanova (1993), who reported that most cells in cat area 17 were optimally excited when a moving visual noise stimulus covered an area extending beyond the classical receptive field. It is also consistent with the idea that V1 neurons may respond to translational Glass pattern stimuli and low-contrast gratings in a similar manner.

\section{DISCUSSION}

Both simple and complex cells in V1 typically gave weak but reliable orientation-selective responses to dynamic, translational Glass patterns. Neuronal selectivity qualitatively matched our predictions from receptive field models on the basis of oriented, linear filters. Orientation selectivity was generally best when the separation of the dots was approximately one-quarter to one-half the optimal spatial period of the receptive field. For larger dot separations $(r \approx \lambda)$, our model predicted and we observed more complicated forms of orientation selectivity in which response peaks $90^{\circ}$ from the optimum were sometimes evident and overall selectivity was reduced. Simulations presented in the Appendix reveal that this behavior should depend on the selectivity of the neuron, being most prominent for neurons with the narrowest orientation and spatial frequency tuning.

If, in fact, the local structure of Glass patterns is detected initially by V1 cells and then pooled in downstream areas, limitations imposed in V1 might be reflected in psychophysical data. Two types of data indicate that this is the case. Psychophysical measurements in humans (Wilson et al., 1997; Wilson and Wilkinson, 1998; Alliston et al., 1999; Ross et al., 2000) and macaque monkeys (McCollum et al., 2000) show that the optimal dot separation for detection of form in Glass patterns is between 0.1 and $0.2^{\circ}$. Recall that our data and simulations show that the best orientation selectivity for a neuron occurred when dot separation was $0.25-0.5$ of the spatial period of the optimal spatial frequency. The range of optimal $r$ values observed behaviorally corresponds to channels or neurons with optimal spatial frequen-

\section{$\leftarrow$}

black dots) differ little in amplitude but significantly in orientation tuning when compared with responses to opposite-polarity patterns (thick black line). An error bar $( \pm 1 \mathrm{SEM})$ is shown at the peak of the oppositepolarity dot tuning curve. $G$, Across a population of 31 cells tested with opposite-polarity patterns, the absolute value of the difference between the preferred grating orientation and the preferred Glass pattern orientation is shown in a frequency histogram. The distribution of differences in optimal orientation is concentrated around $90^{\circ}$ orthogonal to the similar distribution for same-polarity dots (Fig. 4B). 
$\boldsymbol{A}$

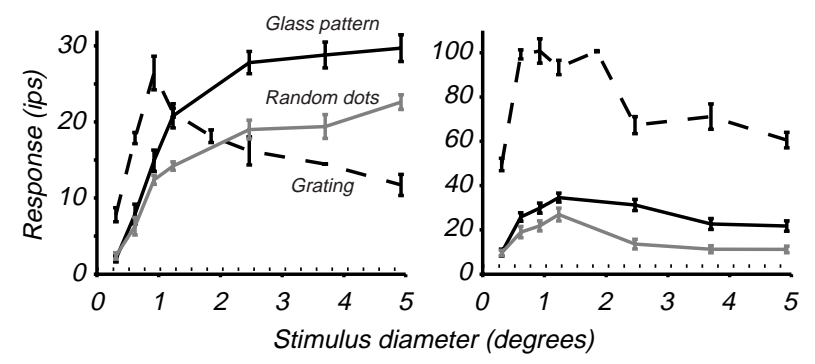

C

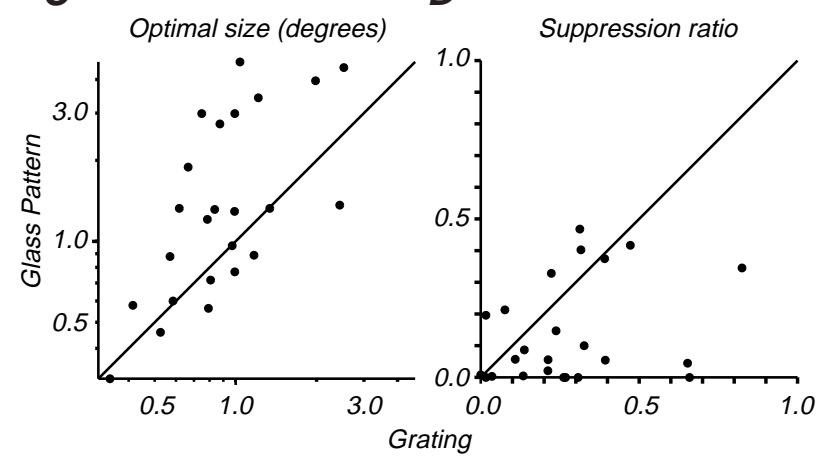

$E$

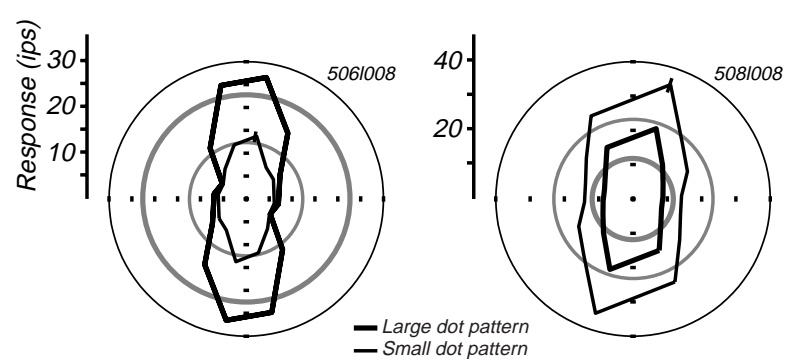

Figure 7. Responses to stimuli of different size. $A, B$, These two cells show suppression for large grating stimuli but differ in their response to dot stimuli of increasing size. The area tuning curves were collected at the optimal orientation and spatial and temporal frequency for gratings. For Glass patterns, the orientation and dot separation were set to be optimal. The cell in $A$ shows an increased response as the dot patterns are increased in size up to $5^{\circ}$, whereas its response peaks to a grating of $\sim 1^{\circ}$. The cell in $B$ is suppressed beyond $\sim 1^{\circ}$ for both dot patterns and gratings. $C$, For most cells, the optimal size for Glass patterns is similar to that for gratings (most points fall near the line of equality). Some cells, like the one in $A$, prefer larger Glass pattern stimuli. On average, the optimal size for Glass pattern stimuli is larger than for gratings. $D$, On average, cells show more suppression for gratings than for Glass patterns. We calculated a suppression ratio, the peak response minus the response at the maximum size, divided by the response at the maximum size. It ranges from zero (for no suppression) to 1 (for complete suppression). Most cells fall near or below the equality line, indicating that suppression for gratings is usually similar to or higher than for Glass patterns. $E, F$, We collected orientation tuning curves for Glass patterns of two sizes (the optimal size for gratings and an extended pattern) in the same two cells. The thick and thin lines indicate response to the large and small dot patterns, respectively. The black lines indicate Glass pattern response, whereas the gray lines indicate the response to random dots. The curves are rotated so that the vector fit of the orientation tuning to gratings is set to zero. An error bar $( \pm 1$ SEM) is shown at the peak of the small Glass pattern tuning curve.

cies between 2.5 and $5 \mathrm{c} / \mathrm{deg}$. This is the range in which both human and monkey observers have their highest contrast sensitivity (Campbell and Robson, 1968; DeValois et al., 1974) and the most common range for the optimal spatial frequencies of macaque cortical neurons representing the central $5^{\circ}$ of the visual field (DeValois et al., 1982). Our V1 neuronal population would thus provide the most accurate information about local orientation of the elements of Glass patterns that have dot separations in precisely the range that is optimal for behavior.

A second psychophysical finding that has been related to the responses of $\mathrm{V} 1$ cells is the relative ineffectiveness of Glass patterns in which the two dots in a pair are of opposite contrast. Glass and Switkes (1976) and Prazdny (1986) have reported that the correct structure cannot be perceived in opposite-polarity Glass patterns. In particular, Glass and Switkes (1976) reported that opposite-polarity concentric patterns appeared "spiral-like." Kovács and Julesz (1992) extended this observation, showing that opposite-polarity Glass patterns elicit reversed perceptions compared with same-polarity patterns (i.e., the perceived orientation is orthogonal to that of the dot pair). These percepts, counterintuitive at the time, were used as evidence to conclude that opposite-polarity Glass patterns did not activate V1 cells. It was suggested that this is consistent with V1 simple cells receiving either on or off inputs, but not both (Hubel and Wiesel, 1962; Tolhurst and Thompson, 1975). However, we have established here that opposite-polarity Glass patterns drive V1 cells (simple and complex) in a manner that can account for the psychophysical results. Furthermore, it is likely that V1 cells receive both on and off inputs in a push-pull manner (for review, see Ferster and Miller, 2000). Our data and simulations both show that neuronal orientation selectivity is reduced for opposite-polarity Glass patterns. V1 neurons would therefore provide a less precise signal about dot-pair orientations with opposite-polarity than with same-polarity dots, which would considerably degrade the ability of downstream neurons to extract global form from oppositepolarity dot patterns.

Other aspects of Glass pattern perception do not have obvious correlates in our V1 data. DeValois and Switkes (1980) reported that adapting to a translational Glass pattern caused a reduction in sensitivity to gratings aligned orthogonal, but not parallel, to the dot-pair orientation. This curious result does not correspond to any simple expected outcome based on the orientation and spatial frequency selectivity of our V1 neurons. In particular, we would expect maximal adaptation for patterns that were parallel, not orthogonal, to the orientation in the adapting pattern. DeValois and Switkes (1980) suggested that the effects they observed might be attributable to a lack of response by cells in V1 caused by inhibitory interactions among neurons with similar preference. Because our V1 cells respond reliably to translational Glass patterns aligned parallel to a preferred grating stimulus, we conclude that the interactions that they propose do not occur at the level of V1.

The links between psychophysical studies of Glass pattern perception and V1 receptive field structure help us to understand the limits of the first stage of form perception, but they cannot provide specific information about downstream stages of analysis that integrate information in Glass patterns to provide signals about global form. Yet, some recent psychophysical and physiological studies do provide clues about this second stage. Wilson and his colleagues (1997) have shown that human observers are more sensitive to concentric Glass patterns than to other types. However, individual V1 receptive fields are not large enough to give selective responses to concentric over translational structure. The particular salience of the concentric Glass pattern must be caused by the action of second-stage mechanisms that selectively pool certain inputs from V1. Neurons in macaque V2 (Hedge and 
Space Domain

$\boldsymbol{A}$

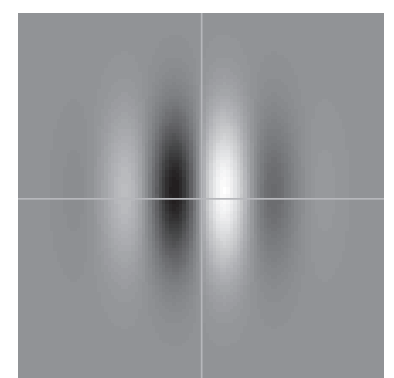

Gabor function ( $\lambda=$ spatial period)

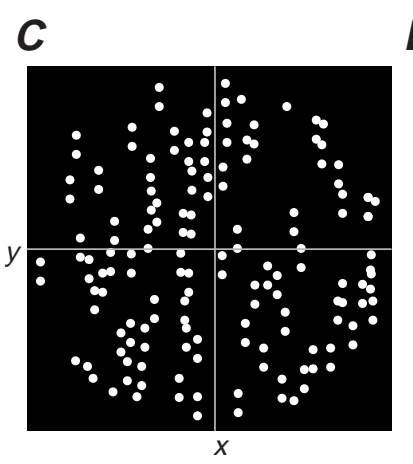

Glass pattern
Frequency Domain

(Power Spectra)

\section{$B$}

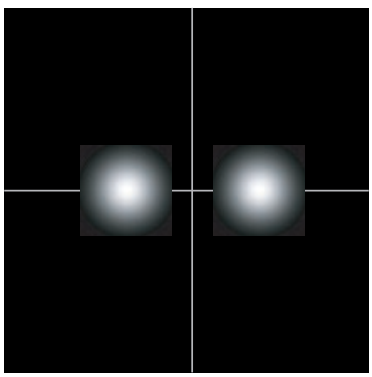

D

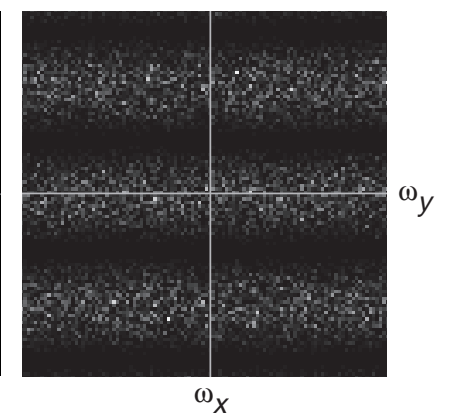

Figure 8. Intuition of Glass pattern tuning from the Fourier domain. The column at left contains representations in the space domain, and the column at right contains the corresponding frequency domain representations (shown as power spectra). In the right column, white indicates an area with higher energy and black indicates lower energy. A, A Gabor function is typically used to model the spatial profile of a V1 simple cell receptive field. The vertical and horizontal axes represent space in the $x$ and $y$ directions, whereas the spatial period of the Gabor is indicated by $\lambda$. $B$, The Fourier transform of a Gabor function is a pair of Gaussian blobs. The orientation, size, and separation of the blobs depend on the characteristics of the Gabor function. $C$, Our experiments used translational Glass patterns, composed of many dot pairs, like the one in $C$. $D$, Because the spatial pattern now contains many dot pairs with random positions, the Fourier representation is a noisy grating.

Van Essen, 2000) and V4 (Gallant et al., 1993, 1996) have been reported to be selective for complex shapes, including concentric and hyperbolic forms. Neurons such as those may form the neural basis for this second stage, and studying their responses to Glass patterns could yield further insight into the processes by which the visual system converts sparse local orientation signals into salient percepts of global form.

\section{APPENDIX}

Before deriving an exact expression for the response of our receptive field model to translational Glass patterns, we will briefly describe a way to intuit the shape of the model's Glass pattern orientation tuning curve in the frequency domain. This approach makes use of the power spectrum of a field of dot pairs, thereby avoiding the position dependence of an individual dot pair, which limited the generality of the demonstration in Figure 2.

The response of a Gabor function (Fig. $8 A$ ) to a stimulus can be determined by multiplying the Fourier transform (FT) of the Gabor function with the FT of the stimulus. The FT of the filter in Figure $8 A$ is a pair of Gaussians in the complex frequency domain, but we will consider only the power spectrum of the filter (the square of the modulus of the FT), which is a real-valued, symmetrical function shown in Figure $8 \mathrm{~B}$. The profile of the power spectrum reveals which spatial frequencies will have the strongest influence on the response of the filter. Next, we visualize the distribution of spatial frequencies in the Glass pattern stimulus (Fig. 8C) with the power spectrum of its FT (Fig. 8D). The power spectrum of a Glass pattern is a noisy sinusoidal grating in which the orientation of the grating depends on the orientation of dot pairs, the wavelength of the grating depends on the dot separation, and the noise is random white-noise determined by the random locations of the dot pairs (here, we do not model the circular aperture of the Glass pattern). For the vertically oriented pattern in $C$, a ridge of elevated power $(D)$ runs horizontally through the regions of sensitivity of the Gabor filter shown in $B$. At this orientation, the stimulus will cause large fluctuations around a mean of zero in the output of the filter, but after rectification these fluctuations will lead to a large positive response. The strength of the response to any Glass pattern stimulus can therefore be estimated by observing how well its power spectrum aligns with that of the Gabor function. If the pattern in $C$ is rotated, its spectrum will rotate, and the bands of high and low power will pass through the sensitive regions of the filter spectrum, yielding a bi-lobed tuning curve. For larger dot separation, the noisy grating in $D$ will have more bands, causing the orientation tuning curve at larger dot separation to have more lobes. Finally, changing the contrast polarity of one dot in the pair shifts the noisy grating in the frequency domain by a quarter cycle. This accounts for the change in orientation tuning with opposite-polarity Glass patterns.

We will now derive an analytical expression for the response of a Gabor patch V1 receptive field to our Glass pattern stimulus as a function of orientation and dot separation. For a simple cell, the neuronal response was modeled as the half-wave rectified output of a linear filter. For a complex cell, the output of two linear filters with a $90^{\circ}$ phase shift were rectified and summed. The receptive field model had an explicit spatial structure, whereas temporal integration was handled implicitly by setting the number of dot pairs that were integrated. Even- and odd-symmetric Gabor patch models, as well as complex and simple cell models, performed in qualitatively the same manner. Below we outline the derivation of the mean simple cell response for any number of dot pairs falling on an arbitrary receptive field profile.

The neuronal receptive field, $f$, was modeled by a Gabor function (a Gaussian $\times$ a sinusoid) as follows:

$$
f(x, y)=e^{-\frac{1}{2}\left(\frac{x^{2}}{\sigma_{w}^{2}}+\frac{y^{2}}{\sigma_{h}^{2}}\right)} \sin (x / \lambda),
$$

where $\sigma_{\mathrm{w}}$ and $\sigma_{\mathrm{h}}$ set the receptive field width and height, $\lambda$ is the preferred spatial period, and the preferred orientation of the receptive field is vertical. One dot pair is represented as a pair of $\delta$-functions:

$$
\Pi(x, y)=\delta(x+\Delta x, y+\Delta y)+\delta(x-\Delta x, y-\Delta y),
$$

where

$$
\begin{aligned}
& \Delta x=\frac{r}{2} \cos (\theta), \\
& \Delta y=\frac{r}{2} \sin (\theta),
\end{aligned}
$$



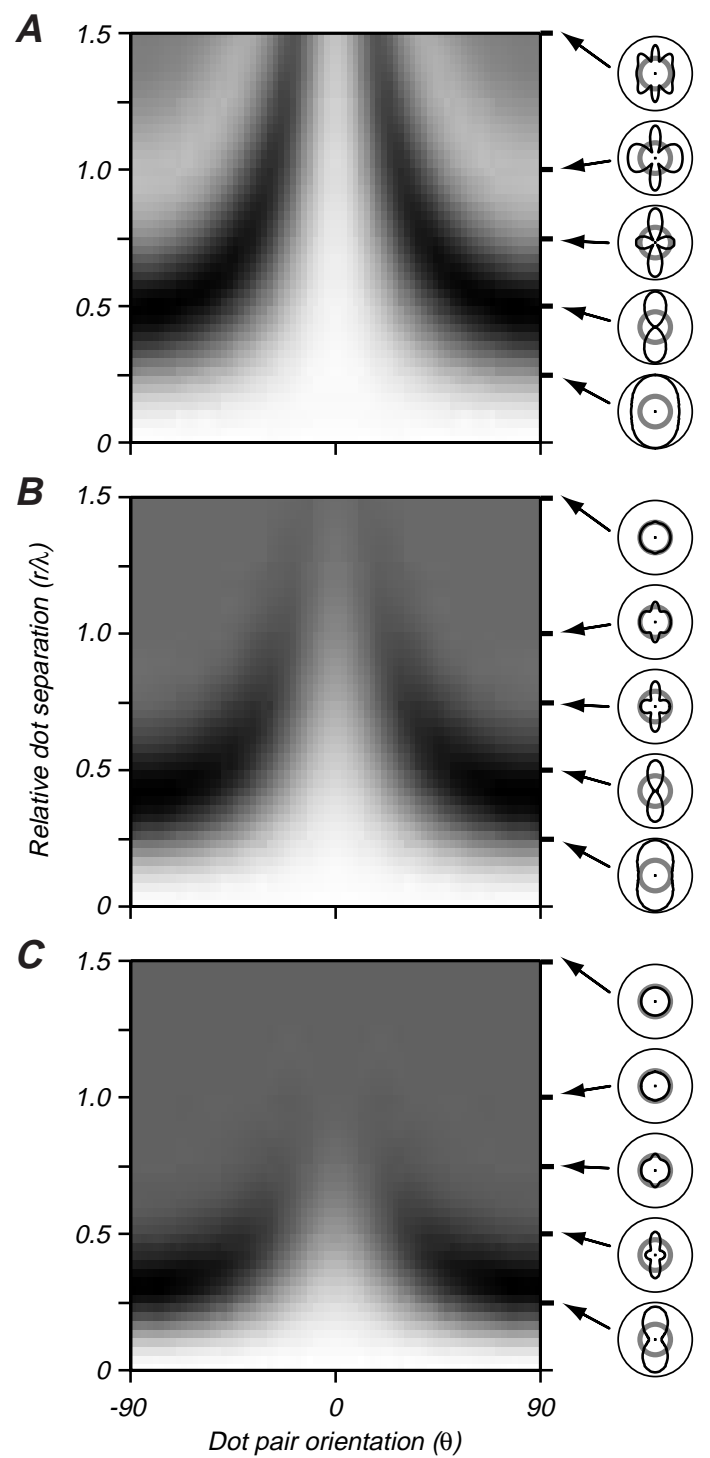

Figure 9. Response surfaces of model response $(r$ vs $\theta)$. The grayscale images in the left column show large responses in white and small responses in black for a range of $r$ and $\theta$. The polar plots in the right column show orientation tuning curves taken at different values of $r(1 / 4,1 / 2,3 / 4$, 1 , and $3 / 2 \times \lambda$, as indicated by the arrows). All three plots are generated from a receptive field with an aspect ratio of 0.6 and frequency of 2.16 $\mathrm{c} / \mathrm{deg}$. $A$, For a receptive field with narrow orientation tuning for gratings (width $=0.32^{\circ}$ ), there is prominent four-lobed tuning when $r=\lambda$. $B$, Model response for a receptive field with typical orientation tuning for gratings (width $=0.16^{\circ}$ ) shows some four-lobed tuning, and the tuning with the highest selectivity and modulation is shifted to lower dot separations. This receptive field is the same as the one used for Figure 2. $C$, For a receptive field with broad orientation tuning for gratings (width $=$ $0.09^{\circ}$ ), there is a complete lack of tuning when $r=\lambda$. The region of highest selectivity and modulation is shifted even lower, near $r=\lambda / 4$.

where $r$ is the dot separation and $\theta$ is the orientation of the line connecting the dots.

The response to a single dot pair falling at location $\left(x_{0}, y_{0}\right)$ on the receptive field is simply the integral of the product $f(x$, $y) \operatorname{II}\left(x-x_{0}, y-y_{0}\right)$; therefore, the response as a function of position for all possible dot-pair locations is given by the convolution:

$$
S_{1}(x, y)=f(x, y) * \Pi(x, y) .
$$

From $S_{1}$, we can compute the probability density function (p.d.f.) for the response to a dot pair that falls randomly within a fixed region that contains the receptive field, $f$. In particular, given a uniform, random choice of the coordinates $\left(x_{\mathrm{i}}, y_{\mathrm{i}}\right)$ from within the stimulus aperture, let the probability that $S_{1}\left(x_{\mathrm{i}}, y_{\mathrm{i}}\right)=s$ be given by the p.d.f. $\rho_{1}^{*}(s)$. The p.d.f. for the response to $n$ dot pairs that fall independently and uniformly within the stimulus aperture is given by the $n$-fold autoconvolution of $\rho_{1}^{*}$, denoted $\rho_{n}^{*}$. For large numbers of dot pairs, application of the central limit theorem leads to an approximation for $\rho_{n}^{*}$ :

$$
\lim _{n \rightarrow \infty} \rho_{n}^{*}=\mathrm{G}(n \mu, \sqrt{n} \sigma)
$$

where $\mu$ and $\sigma$ are the mean and SD of $\rho_{1}^{*}$, and $\mathrm{G}$ is the Gaussian p.d.f. The rectification stage maps negative firing rates to zero, so the p.d.f. for the final neuronal response is:

$$
\rho_{n}(s)= \begin{cases}0 & \text { for } s<0 \\ \delta(0) \int_{-\infty}^{0} \rho_{n}^{*}(\phi) d \phi & \text { for } s=0 \\ \rho_{n}^{*}(s) & \text { otherwise. }\end{cases}
$$

The expected value, $M$, of the neuronal response for all $r$ and $\theta$ is then given by:

$$
M(r, \theta)=\int_{0}^{\infty} s \rho_{n}(s ; r, \theta) d s,
$$

where the notation $\rho_{\mathrm{n}}(s ; r, \theta)$ indicates $\rho_{\mathrm{n}}$ computed for a particular value of $r$ and $\theta$. This function is simple to compute numerically for any spatial receptive field, $f$, that goes to zero beyond some finite region. Because a Gaussian extends indefinitely, we truncated our Gabor receptive fields beyond 3 SDs from the center.

Figure 9 shows $M$ plotted for three different model receptive fields (see legend for parameters). Receptive fields in macaque V1 show a wide range of selectivity for orientation and spatial frequency (DeValois et al., 1982). The three receptive fields were designed to represent the upper and lower extremes of this range (Fig. 9A,C) and its average (Fig. 9B). The left column contains grayscale images that represent response strength: white shows high response and black shows low response. These plots can be interpreted as vertically stacked families of orientation tuning curves, parametric in dot separation. The right column shows polar plots of orientation tuning (similar to those in Fig. $2 C-F$ ) taken at different dot separations. These three model plots and the corresponding slices illustrate the variation in Glass pattern tuning across a plausible range of receptive field geometries. In cells with narrow orientation tuning, there is the potential for four-lobed tuning when $r \approx \lambda$ (Fig. 9A). Typical cells (Fig. 9B) show little or no tuning when $r=\lambda$, but have their strongest tuning when $r \approx \lambda / 2$. Cells with broad tuning (Fig. 9C) show a similar lack of strong Glass pattern tuning.

\section{REFERENCES}

Alliston EL, Landy MS, Movshon JA (1999) Detection of form and motion in dynamic Glass patterns and optic flow stimuli. Invest Ophthalmol Vis Sci 40/4:4041.

Alliston EL, Friehling MS, Smith MA, Landy MS, Movshon JA (2001) Detectability of global form in Glass patterns and random dot motion stimuli depends only on signal-to-noise ratio. Invest Ophthalmol Vis Sci 42/4:4683. 
Blakemore C, Tobin E (1972) Lateral inhibition between orientation detectors in the cat's visual cortex. Exp Brain Res 15:439-440.

Campbell FW, Robson JG (1968) Application of Fourier analysis to the visibility of gratings. J Physiol (Lond) 197:551-566.

Carandini M, Heeger DJ, Movshon JA (1997) Linearity and normalization in simple cells of the macaque primary visual cortex. J Neurosci 17:8621-8644.

Casanova C (1993) Responses of cells in cat's area 17 to random dot patterns: influence of stimulus size. NeuroReport 4:1011-1014.

Cavanaugh JR, Bair W, Movshon JA (2002) Nature and interaction of signals from the receptive field center and surround in macaque V1 neurons. J Neurophysiol, in press.

Dakin SC (1997a) The detection of structure in Glass patterns: psychophysics and computational models. Vision Res 37:2227-2246.

Dakin SC (1997b) Glass patterns: some contrast effects re-evaluated. Perception 26:253-268.

Dakin SC, Bex PJ (2001) Local and global visual grouping: tuning for spatial frequency and contrast. J Vision 1:99-111. http://journalof vision. org $/ 1 / 2 / 4$.

DeAngelis GC, Freeman RD, Ohzawa I (1994) Length and width tuning of neurons in the cat's primary visual cortex. J Neurophysiol 71: 347-374.

DeValois KK, Switkes E (1980) Spatial frequency interaction of dot patterns and gratings. Proc Natl Acad Sci USA 77:662-665.

DeValois RL, Morgan H, Snodderly DM (1974) Psychophysical studies of monkey vision. III. Spatial luminance contrast sensitivity tests of macaque and human observers. Vision Res 14:75-81.

DeValois RL, Albrecht DG, Thorell LG (1982) Spatial frequency selectivity of cells in macaque visual cortex. Vision Res 22:545-559.

DeValois RL, Thorell LG, Albrecht DG (1985) Periodicity of striatecortex-cell receptive fields. J Opt Soc Am A 2:1115-1123.

Earle DC (1985) Perception of Glass pattern structure with stereopsis. Perception 14:545-552.

Ferster D, Miller KD (2000) Neural mechanisms of orientation selectivity in the visual cortex. Annu Rev Neurosci 23:441-471.

Foster KH, Gaska JP, Nagler M, Pollen DA (1985) Spatial and temporal frequency selectivity of neurones in visual cortical areas V1 and V2 of the macaque monkey. J Physiol (Lond) 365:331-363.

Gallant JL, Braun J, Van Essen DC (1993) Selectivity for polar, hyperbolic, and cartesian gratings in macaque visual cortex. Science 259: 100-103.

Gallant JL, Connor CE, Rakshit S, Lewis JW, Van Essen DC (1996) Neural responses to polar, hyperbolic, and cartesian gratings in area V4 of the macaque monkey. J Neurophysiol 76:2718-2739.

Glass L (1969) Moire effect from random dots. Nature 223:578-580.

Glass L, Perez R (1973) Perception of random dot interference patterns. Nature 246:360-362.

Glass L, Switkes E (1976) Pattern recognition in humans: correlations which cannot be perceived. Perception 5:67-72.

Hedge J, Van Essen DC (2000). Selectivity for complex shapes in primate visual area V2. J Neurosci 20:RC61.

Hubel D, Wiesel T (1962) Receptive fields, binocular interaction and functional architecture in the cat's visual cortex. J Physiol (Lond) 160:106-154.

Hubel D, Wiesel T (1968) Receptive fields and functional architecture of monkey striate cortex. J Physiol (Lond) 195:215-243.

Jones JP, Palmer LA (1987) An evaluation of the two-dimensional Ga- bor filter model of simple receptive fields in cat striate cortex. J Neurophysiol 58:1233-1258.

Knierim JJ, Van Essen DC (1992) Neuronal responses to static texture patterns in area V1 of the alert macaque monkey. J Neurophysiol 67:961-980.

Kovács I, Julesz B (1992) Depth, motion, and static-flow perception at metaisoluminant color contrast. Proc Natl Acad Sci USA 89: 10390-10394.

Leventhal AG, Thompson KG, Lui D, Zhou Y, Ault SJ (1995) Concomitant sensitivity to orientation, direction, and color of cells in layers 2 , 3 , and 4 of monkey striate cortex. J Neurosci 15:1808-1818.

Levick WR, Thibos LN (1982) Analysis of orientation bias in cat retina. J Physiol (Lond) 329:243-261.

Levitt JB, Kiper DC, Movshon JA (1994) Receptive fields and functional architecture of macaque V2. J Neurophysiol 71:2517-2542.

Marčelja S (1980) Mathematical description of the responses of simple cortical cells. J Opt Soc Am A 70:1297-1300.

McCollum JF, Tang C, Tambar K, Alliston EL, Kiorpes L, Movshon JA (2000) Contrast and luminance dependence of global motion perception in macaques and humans. Soc Neurosci Abstr 26:447.

Merrill EG, Ainsworth A (1972) Glass-coated platinum-plated tungsten microelectrode. Med Biol Eng 10:495-504.

Movshon JA, Thompson ID, Tolhurst DJ (1978a) Spatial summation in the receptive fields of simple cells in the cat's striate cortex. J Physiol (Lond) 283:53-77.

Movshon JA, Thompson ID, Tolhurst DJ (1978b) Receptive field organization of complex cells in the cat's striate cortex. J Physiol (Lond) 283:79-99.

Nelson JI, Frost BJ (1978) Orientation-selective inhibition from beyond the classical visual receptive field. Brain Res 139:359-365.

Newsome WT, Britten KH, Movshon JA (1989) Neuronal correlates of a perceptual decision. Nature 341:52-54.

O'Keefe LP, Movshon JA (1998) Processing of first- and second-order motion signals by neurons in area MT of the macaque monkey. Vis Neurosci 15:305-317.

Parker AJ, Hawken MJ (1988) Two-dimensional spatial structure of receptive fields in monkey striate cortex. J Opt Soc Am A 5:598-605.

Poggio GF, Motter BC, Squatrito S, Trotter Y (1985) Responses of neurons in visual cortex (V1 and V2) of the alert macaque to dynamic random-dot stereograms. Vision Res 25:397-406.

Prazdny K (1984) On the perception of Glass patterns. Perception 13:469-478.

Prazdny K (1986) Some new phenomena in the perception of Glass patterns. Biol Cybern 53:153-158.

Ross J, Badcock D, Hayes A (2000) Coherent global motion in the absence of coherent velocity signals. Curr Biol 10:679-682.

Sceniak MP, Ringach DL, Hawken MJ, Shapley R (1999) Contrast's effect on spatial summation by macaque V1 neurons. Nat Neurosci 2:733-739.

Skottun BC, DeValois RL, Grosof DH, Movshon JA, Albrecht DG, Bonds AB (1991) Classifying simple and complex cells on the basis of response modulation. Vision Res 31:1079-1086.

Tolhurst D, Thompson P (1975) Orientation illusions and aftereffects: inhibition between channels. Vision Res 5:967-972.

Wilson HR, Wilkinson F (1998) Detection of global structure in Glass patterns: implications for form vision. Vision Res 38:2933-2947.

Wilson HR, Wilkinson F, Asaad W (1997) Concentric orientation summation in human form vision. Vision Res 37:2325-2330. 